\title{
REFINED INERTIALLY AND SPECTRALLY ARBITRARY ZERO-NONZERO PATTERNS*
}

\author{
L. DEAETT ${ }^{\dagger}$, D.D. OLESKY ${ }^{\ddagger}$, AND P. VAN DEN DRIESSCHE ${ }^{\dagger}$
}

\begin{abstract}
The refined inertia of a matrix is a quadruple specifying its inertia and additionally the number of its eigenvalues equal to zero. Spectral properties, especially the refined inertias, of real matrices with a given zero-nonzero pattern are investigated. It is shown that every zero-nonzero refined inertially arbitrary pattern of order 4 or less is also spectrally arbitrary. Irreducible and reducible examples are presented to show that for higher orders this is not the case. A further example shows that two zero-nonzero patterns that are not refined inertially arbitrary can have a direct sum that is refined inertially arbitrary, paralleling a known result for inertially arbitrary patterns. Analogously, it is shown that the direct sum of two zero-nonzero patterns may be spectrally arbitrary even if neither summand is spectrally arbitrary.
\end{abstract}

Key words. Zero-nonzero pattern, Refined inertia, Inertially arbitrary, Spectrally arbitrary.

AMS subject classifications. 15B35, 15A18, 05C50.

1. Introduction. The placement of the zero and nonzero entries in a matrix describes its combinatorial structure. Properties of this structure often have important implications, for example when the matrix in question is derived from a linearized dynamical system (see, e.g., [11], where the signs of the nonzero entries are also specified). The main purpose of this paper is to consider how this combinatorial description relates to spectral properties of real matrices with that structure, especially in terms of refined inertia (as defined below). To specifically describe these structural and spectral properties, we state some definitions.

A zero-nonzero pattern is a matrix with entries from the set $\{0, *\}$. When unambiguous, we use the term pattern alone to refer to a zero-nonzero pattern. Given a real matrix $A$, if $\mathcal{A}$ is obtained by replacing every nonzero entry of $A$ with a $*$, then $A$ has pattern $\mathcal{A}$. A pattern $\mathcal{A}^{\prime}$ is a superpattern of $\mathcal{A}$ if $\mathcal{A}$ can be obtained by replacing some (possibly empty) subset of the nonzero entries of $\mathcal{A}^{\prime}$ with zeros. Two square patterns $\mathcal{A}$ and $\mathcal{B}$ are equivalent if one can be obtained from the other by any combination of transposition and permutation similarity. A square pattern is

*Received by the editors March 23, 2010. Accepted for publication July 31, 2010. Handling Editor: Michael Tsatsomeros.

${ }^{\dagger}$ Dept of Mathematics and Statistics, University of Victoria, P.O. Box 3060, Victoria, BC, Canada V8W 3R4 (deaett@math.uvic.ca, pvdd@math.uvic.ca).

${ }^{\ddagger}$ Dept of Computer Science, University of Victoria, P.O. Box 3055, Victoria, BC, Canada V8W 3P6 (dolesky@cs.uvic.ca). 
reducible if it is permutation similar to a pattern of the form $\left[\begin{array}{ll}\mathcal{X} & \mathcal{Y} \\ O & \mathcal{Z}\end{array}\right]$, where $\mathcal{X}$ and $\mathcal{Z}$ are square and non-vacuous. A pattern that is not reducible is irreducible.

The inertia of a matrix $A$ of order $n$ is the ordered triple $\left(n_{+}, n_{-}, n_{0}\right)$ of nonnegative integers that sum to $n$, where $n_{+}$is the number of eigenvalues of $A$ with positive real part, $n_{-}$is the number with negative real part, and $n_{0}$ is the number with zero real part. A pattern $\mathcal{A}$ allows a given inertia if there is some matrix $A$ having both this inertia and the pattern $\mathcal{A}$. If a pattern $\mathcal{A}$ allows every possible inertia, then $\mathcal{A}$ is an inertially arbitrary pattern (IAP). Recent results concerning inertially arbitrary patterns appear, for example, in $[3,4,8,10]$.

A pattern $\mathcal{A}$ allows characteristic polynomial $p(x)$ if there is some matrix $A$ that has both characteristic polynomial $p(x)$ and the pattern $\mathcal{A}$. If a pattern $\mathcal{A}$ of order $n$ allows every monic polynomial of degree $n$ with real coefficients, then $\mathcal{A}$ is a spectrally arbitrary pattern (SAP). The concept of a spectrally arbitrary pattern was introduced in [7] for sign patterns, where a sign pattern is a matrix with entries from the set $\{+,-, 0\}$. The notions of superpattern, reducible, irreducible, allow, and inertially and spectrally arbitrary are defined analogously for sign patterns as they are for zero-nonzero patterns.

In this paper, we concentrate on zero-nonzero patterns, but our motivation is derived from questions that have been posed for sign patterns. In particular, we use the notion of refined inertia that was introduced in [9] for sign patterns.

Let $A$ be a real matrix of order $n$. The refined inertia of $A$ is the ordered quadruple $\left(n_{+}, n_{-}, n_{z}, 2 n_{p}\right)$ of nonnegative integers that sum to $n$, where $\left(n_{+}, n_{-}, n_{z}+2 n_{p}\right)$ is the inertia of $A$ while $n_{z}$ is the algebraic multiplicity of zero as an eigenvalue of $A$, and $2 n_{p}$ is the number of nonzero pure imaginary eigenvalues. Thus, in the refined inertia, the number of eigenvalues with zero real part ( $n_{0}$ in the inertia) has been split into $n_{z}$ and $2 n_{p}$, a split that may have important consequences when the pattern in question is derived from a linearized dynamical system. A pattern $\mathcal{A}$ allows refined inertia $\left(n_{+}, n_{-}, n_{z}, 2 n_{p}\right)$ if there is some matrix having both this refined inertia and the pattern $\mathcal{A}$. If a pattern $\mathcal{A}$ allows every possible refined inertia, then $\mathcal{A}$ is a refined inertially arbitrary pattern (rIAP), and this notion is defined analogously for sign patterns.

The reversal of a refined inertia is obtained by exchanging the first two entries in the quadruple; i.e. the reversal of $\left(n_{+}, n_{-}, n_{z}, 2 n_{p}\right)$ is $\left(n_{+}^{\prime}, n_{-}^{\prime}, n_{z}, 2 n_{p}\right)$, where $n_{+}^{\prime}=n_{-}$and $n_{-}^{\prime}=n_{+}$. Note that, as $A$ and $-A$ have the same zero-nonzero pattern, a pattern $\mathcal{A}$ allows a given refined inertia if and only if it allows the reversal of that refined inertia. Hence, in order to verify that a given pattern is an rIAP, we need to verify only that it allows one of each possible refined inertia and its reversal. 
For $n \geq 2$, the maximum number of distinct inertias allowed by any zero-nonzero or sign pattern of order $n$ is easily seen to be $(n+1)(n+2) / 2=\left(\begin{array}{c}n+2 \\ 2\end{array}\right)$. Here we derive a formula for the maximum number of distinct refined inertias allowed by any zero-nonzero or sign pattern of order $n \geq 2$. (A zero-nonzero pattern of order 1 allows either 1 or 2 refined inertias.)

THEOREM 1.1. The maximum number of distinct refined inertias allowed by any zero-nonzero or sign pattern of order $n \geq 2$ is

$$
\begin{array}{lr}
\frac{\left(\frac{n}{2}+1\right)\left(\frac{n}{2}+2\right)(2 n+3)}{6} & \text { for } n \text { even, and } \\
\frac{\left\lceil\frac{n}{2}\right\rceil\left(\left\lceil\frac{n}{2}\right\rceil+1\right)\left(4\left\lceil\frac{n}{2}\right\rceil+5\right)}{6} & \text { for } n \text { odd } .
\end{array}
$$

The maximum number of distinct refined inertias, excluding reversals, allowed by any zero-nonzero pattern of order $n \geq 2$ is

$$
\begin{array}{lr}
\frac{(n+2)(n+3)(n+4)}{24} & \text { for } n \text { even, and } \\
\frac{(n+1)(n+3)(n+5)}{24} & \text { for } n \text { odd } .
\end{array}
$$

Proof. For $n \geq 2$, let $R(n)$ denote the maximum number of distinct refined inertias allowed by any pattern of order $n$. It is trivial to check that $R(2)=7$ and $R(3)=13$, in agreement with the formulas given above.

Suppose $n \geq 4$. If $n$ is even, say $n=2 k$, then there are $\left(\begin{array}{c}2 k+2 \\ 2\end{array}\right)$ possible refined inertias with $n_{p}=0$ and $R(2 k-2)$ possible refined inertias with $n_{p}>0$. Hence, by induction,

$$
\begin{aligned}
R(n) & =\left(\begin{array}{c}
2 k+2 \\
2
\end{array}\right)+R(2 k-2) \\
& =\frac{(2 k+2)(2 k+1)}{2}+\frac{k(k+1)(4 k-1)}{6} \\
& =\frac{(k+1)(k+2)(4 k+3)}{6}
\end{aligned}
$$

and the proof is complete as $k=\frac{n}{2}$. If $n$ is odd, say $n=2 k+1$, then similarly,

$$
\begin{aligned}
R(n) & =\left(\begin{array}{c}
2 k+1+2 \\
2
\end{array}\right)+R(2 k-1) \\
& =\frac{(2 k+3)(2 k+2)}{2}+\frac{k(k+1)(4 k+5)}{6} \\
& =\frac{(k+1)(k+2)(4(k+1)+5)}{6}
\end{aligned}
$$


and the proof is complete as $k+1=\left\lceil\frac{n}{2}\right\rceil$.

By definition, these maximum values are achieved by any rIAP of order $n$. In particular, any full pattern (i.e., a pattern with all entries $*$ ) of order $n \geq 2$ is a SAP (see e.g., [2]) and hence is an rIAP.

Now let $\tilde{R}(n)$ denote the maximum number of distinct refined inertias, excluding reversals, allowed by any pattern of order $n \geq 2$. It is trivial to check that $\tilde{R}(2)=5$ and $\tilde{R}(3)=8$, in agreement with the formulas given above.

Suppose $n \geq 4$. If $n$ is even, say $n=2 k$, the number of possible refined inertias in which the first two coordinates are both equal to $i$ is $k-i+1$, for $0 \leq i \leq k$. Hence there are $\sum_{i=0}^{k}(k-i+1)=\frac{(k+1)(k+2)}{2}$ refined inertias that are equal to their own reversals. Thus

$$
\tilde{R}(n)=\frac{1}{2}\left(R(n)-\frac{(k+1)(k+2)}{2}\right)+\frac{(k+1)(k+2)}{2}=\frac{(2 k+2)(2 k+3)(2 k+4)}{24}
$$

and the proof is complete as $2 k=n$. The proof for $n$ odd is similar.

For a pattern $\mathcal{X}$ of order $n$, let $D(\mathcal{X})$ be the directed graph on vertices $\left\{v_{1}, \ldots, v_{n}\right\}$ in which an arc is present from $v_{i}$ to $v_{j}$ if and only if the $(i, j)$ entry of $\mathcal{X}$ is nonzero. In analyzing the refined inertias or characteristic polynomials allowed by a particular pattern, the following result of [8] (the sign pattern version of which appeared earlier in [1]) is used to normalize matrices throughout the remainder of this paper.

Lemma 1.2 ([8, Proposition 2]). Let $A$ be a matrix of order $n$ with irreducible zero-nonzero pattern $\mathcal{A}$. If $T$ is a directed subgraph of $D(\mathcal{A})$ and the underlying (undirected) graph of $T$ is a tree, then $A$ is diagonally similar to a matrix with the same zero-nonzero pattern in which all entries corresponding to arcs of $T$ are 1.

In [9] the question was raised as to whether or not a sign pattern that is an rIAP must be a SAP. In Section 2 we address this question for irreducible zero-nonzero patterns and exhibit such a pattern of smallest order that is an rIAP but not a SAP. Section 3 focuses on reducible zero-nonzero patterns. There we exhibit a direct sum of smallest order that is an rIAP but not a SAP, and a smallest order direct sum of two zero-nonzero patterns that is an rIAP with neither summand an rIAP. This latter example is of order 8, one less than the order of a smallest direct sum of two zerononzero non-IAPs that is an IAP as given in [3, Example 9]. In Section 4, we address an open question stated in [3] by giving an example of two zero-nonzero patterns with neither a SAP such that their direct sum is a SAP.

2. Refined inertia and irreducible nonzero patterns. We first investigate the relationship between the refined inertias allowed by a given pattern and the characteristic polynomials allowed by the pattern. We begin by showing that for matrices 
of order at most 4, a pattern that allows all refined inertias must allow all characteristic polynomials.

Spectral properties of the following pattern are important in most of our results.

Definition 2.1. Let

$$
\mathcal{M}=\left[\begin{array}{llll}
* & * & * & 0 \\
* & * & * & 0 \\
0 & 0 & 0 & * \\
* & * & 0 & 0
\end{array}\right]
$$

In [6] a particular signing of $\mathcal{M}$ was analyzed in detail, and the following result was obtained, which we state here in the weaker case of the associated zero-nonzero pattern.

Lemma 2.2 ([6, Corollary 2.3]). Let $p(x)$ be a monic polynomial of degree 4 with real coefficients. Each of the following conditions is sufficient for $\mathcal{M}$ to allow the characteristic polynomial $p(x)$.

(i) The constant term of $p(x)$ is positive.

(ii) The zeros of $p(x)$ are all real and the constant term of $p(x)$ is zero.

The conditions provided by Lemma 2.2 for a characteristic polynomial to be allowed by $\mathcal{M}$ are sufficient to determine precisely which refined inertias are allowed by $\mathcal{M}$.

Proposition 2.3. The pattern $\mathcal{M}$ does not allow $(0,0,2,2)$ but does allow every other refined inertia.

Proof. Note that $\mathcal{M}$ allows the refined inertia $(0,0,2,2)$ if and only if it allows some characteristic polynomial of the form $x^{4}+q x^{2}$ for $q>0$, which we now show is not the case.

Suppose $A$ is a matrix with pattern $\mathcal{M}$. Without loss of generality,

$$
A=\left[\begin{array}{llll}
a & 1 & 1 & 0 \\
b & c & d & 0 \\
0 & 0 & 0 & 1 \\
e & f & 0 & 0
\end{array}\right]
$$

for some nonzero real numbers $a, b, c, d, e$ and $f$. Then the characteristic polynomial of $A$ is

$$
p_{A}(x)=x^{4}+(-a-c) x^{3}+(a c-b) x^{2}+(-d f-e) x+(a d f-b f+c e-d e) .
$$


Suppose $p_{A}(x)=x^{4}+q x^{2}$. Then $a=-c$ while $e=-d f$ so that

$$
p_{A}(x)=x^{4}+\left(-c^{2}-b\right) x^{2}+\left(-2 c d f-b f+d^{2} f\right) .
$$

That $q$ is the coefficient of $x^{2}$ implies $b=-q-c^{2}$ and hence

$$
p_{A}(x)=x^{4}+q x^{2}+\left((c-d)^{2}+q\right) f .
$$

But then, as $f$ is nonzero, a zero constant term requires that $q \leq 0$. Hence, $\mathcal{M}$ cannot allow any characteristic polynomial of the form $x^{4}+q x^{2}$ for $q>0$ and therefore does not allow the refined inertia $(0,0,2,2)$.

The fact that $\mathcal{M}$ allows every other refined inertia follows from Lemma 2.2, as it is straightforward to exhibit a polynomial that has any one of the other 13 (excluding reversals) refined inertias.

Definition 2.4. Let

$$
\mathcal{T}_{2}=\left[\begin{array}{ll}
* & * \\
* & *
\end{array}\right] .
$$

This pattern $\mathcal{T}_{2}$ is the zero-nonzero pattern associated with the sign pattern of order 2 belonging to a family of tridiagonal sign patterns introduced in [7]. Every pattern of that family is known to be a SAP for $n \leq 16$ (see [2] for details), and in particular the zero-nonzero pattern $\mathcal{T}_{2}$ is a SAP.

THEOREM 2.5. For $n \leq 4$, a zero-nonzero pattern of order $n$ is an rIAP if and only if it is a SAP.

Proof. For $n=1$ the statement is vacuously true. For $n=2$ it is shown in [4] that $\mathcal{T}_{2}$ is the only IAP, and hence the only possible rIAP. Since $\mathcal{T}_{2}$ is a SAP, the proof is complete in this case. For $n=3$, the zero-nonzero IAPs are classified by [4, Proposition 2.2] and are SAPs by [5, Theorem 1.1]. Hence, for $n \leq 3$, a pattern is an IAP if and only if it is an rIAP if and only if it is a SAP.

The case $n=4$ requires some analysis. If a zero-nonzero rIAP of order 4 is reducible, then by [4, Proposition 2.3] it is equivalent to a reducible superpattern of $\mathcal{T}_{2} \oplus \mathcal{T}_{2}$ and hence is a SAP (see e.g. [6, Proposition 2.1]). If a zero-nonzero IAP of order 4 is irreducible, then it follows from [4, Proposition 2.4 and Theorem 2.5] that the pattern must be either a SAP or equivalent to one of

$$
\mathcal{N}_{1}^{*}=\left[\begin{array}{cccc}
* & * & 0 & 0 \\
0 & 0 & * & * \\
* & * & 0 & 0 \\
0 & 0 & * & *
\end{array}\right], \mathcal{N}_{2}^{*}=\left[\begin{array}{cccc}
* & * & * & 0 \\
* & * & * & 0 \\
0 & 0 & 0 & * \\
* & * & 0 & 0
\end{array}\right] \text { or } \mathcal{N}_{3}^{*}=\left[\begin{array}{cccc}
* & * & * & 0 \\
* & * & * & 0 \\
* & 0 & 0 & * \\
* & 0 & 0 & 0
\end{array}\right]
$$


Hence, it remains to show that none of these three patterns is an rIAP.

The pattern $\mathcal{N}_{1}^{*}$ is not an rIAP as it does not allow the refined inertia $(0,0,0,4)$. To see this, note that if $A$ has the pattern $\mathcal{N}_{1}^{*}$, then without loss of generality

$$
A=\left[\begin{array}{llll}
a & 1 & 0 & 0 \\
0 & 0 & 1 & 1 \\
b & c & 0 & 0 \\
0 & 0 & d & e
\end{array}\right]
$$

and the characteristic polynomial of $A$ is

$$
p_{A}(x)=x^{4}+(-a-e) x^{3}+(a e-c) x^{2}+(a c-c d+c e-b) x+(d-e)(c a-b) .
$$

If $A$ has refined inertia $(0,0,0,4)$, then

$$
p_{A}(x)=\left(x^{2}+\alpha\right)\left(x^{2}+\beta\right)=x^{4}+(\alpha+\beta) x^{2}+\alpha \beta
$$

for some $\alpha>0$ and $\beta>0$. Hence $a=-e$ and $b=a c-c d+c e=-c d$. Also $c=a e-\alpha-\beta=-e^{2}-\alpha-\beta$, so that $c<0$. But now the constant term is

$$
(d-e)(a c-b)=(d-e)((-e) c-(-c d))=(d-e)(-c e+c d)=c(d-e)^{2} \leq 0,
$$

while the constant term is also $\alpha \beta>0$, a contradiction.

The pattern $\mathcal{N}_{2}^{*}$ is equal to $\mathcal{M}$ (Definition 2.1) and hence by Proposition 2.3 is not an rIAP.

The pattern $\mathcal{N}_{3}^{*}$ is not an rIAP as it does not allow the refined inertia $(0,0,4,0)$. To see this, note that if $A$ has the pattern $\mathcal{N}_{3}^{*}$, then without loss of generality

$$
A=\left[\begin{array}{llll}
a & 1 & b & 0 \\
c & d & 1 & 0 \\
e & 0 & 0 & 1 \\
f & 0 & 0 & 0
\end{array}\right]
$$

and the characteristic polynomial of $A$ is

$$
p_{A}(x)=x^{4}+(-a-d) x^{3}+(a d-b e-c) x^{2}+(b d e-b f-e) x+(b d-1) f .
$$

If $A$ has refined inertia $(0,0,4,0)$, then every coefficient of $p(x)$ is zero. Thus $b d=1$ and hence the coefficient of $x$ is $b d e-b f-e=e-b f-e=-b f$. But this last quantity cannot be zero with $b$ and $f$ both nonzero.

The proof of Theorem 2.5 makes use of the fact that, for patterns of orders 2 and 3 , the notions of IAP, rIAP and SAP are equivalent. The proof also shows that, for a 
pattern of order 4, being an IAP no longer implies being an rIAP; however being an rIAP is still equivalent to being a SAP, as the theorem states. We now show that for patterns of order 5, being an rIAP no longer implies being a SAP. Consequently, no two of the notions of IAP, rIAP and SAP are equivalent.

We now introduce the irreducible zero-nonzero pattern $\mathcal{L}$ of order 5 that was discovered by considering superpatterns of the pattern $\mathcal{K}$ from [10]. This pattern $\mathcal{L}$ plays a leading role in two of our main results.

DEFINITION 2.6. Let

$$
\mathcal{L}=\left[\begin{array}{lllll}
* & * & 0 & 0 & * \\
0 & 0 & * & 0 & * \\
0 & 0 & 0 & * & 0 \\
0 & 0 & * & 0 & * \\
* & * & 0 & 0 & *
\end{array}\right]
$$

The following result precisely captures which characteristic polynomials are allowed by $\mathcal{L}$.

Proposition 2.7. Let $p(x)=x^{5}+p_{4} x^{4}+p_{3} x^{3}+p_{2} x^{2}+p_{1} x+p_{0}$. Then $\mathcal{L}$ fails to allow characteristic polynomial $p(x)$ if and only if $p_{4}=p_{2}=0$ while $p_{0} \neq 0$.

Proof. If $A$ has pattern $\mathcal{L}$, then without loss of generality

$$
A=\left[\begin{array}{lllll}
a & 1 & 0 & 0 & b \\
0 & 0 & 1 & 0 & h \\
0 & 0 & 0 & 1 & 0 \\
0 & 0 & c & 0 & 1 \\
d & f & 0 & 0 & g
\end{array}\right]
$$

for some nonzero real numbers $a, b, c, d, f, g$ and $h$ and has characteristic polynomial

$$
\begin{aligned}
p_{A}(x)=x^{5}-(a+g) x^{4}+(a g-b d & -f h-c) x^{3}+(c(a+g)-h(d-f a)) x^{2} \\
& +(c f h-f-a c g+b c d) x+(d-f a)(c h-1) .
\end{aligned}
$$

Thus if $p_{A}(x)=p(x)$ with $p_{4}=p_{2}=0$, then $a+g=0$ and $d-f a=0$, implying that the constant term of $p(x)$ must be zero as well. Hence if $p_{4}=p_{2}=0$ while $p_{0} \neq 0$, then $\mathcal{L}$ fails to allow $p(x)$.

For the other direction, assume that $p_{4} \neq 0$ or $p_{2} \neq 0$ or $p_{0}=0$. Let $a, b, c, d, f$, $g$ and $h$ be chosen as follows. First let the following four polynomials be defined for 
any real number $a$.

$$
\begin{aligned}
& q_{1}(t)=a p_{4}\left(a+p_{4}\right) t^{2}+\left(p_{0}+a^{2} p_{2}-p_{1} p_{4}+a p_{2} p_{4}\right) t+\left(a^{2} p_{0}-p_{1} p_{2}+p_{0} p_{3}+a p_{0} p_{4}\right) \\
& q_{2}(t)=p_{4} t^{2}+p_{2} t+p_{0} \\
& q_{3}(t)=\left(a+p_{4}\right) t^{2}+\left(a p_{3}+p_{2}\right) t+\left(p_{0}+a p_{1}\right) \\
& q_{4}(t)=t^{2}+p_{3} t+p_{1}
\end{aligned}
$$

Case 1. $p_{4} \neq 0$ or $p_{2} \neq 0$. Choose any nonzero $a$ with $a \neq-p_{4}$. It is then possible to choose $c \neq 0$ such that $q_{1}(c), q_{2}(c), q_{3}(c)$ and $q_{4}(c)$ are all nonzero while $p_{2}+c p_{4} \neq 0$. Then take

$$
b=\frac{q_{1}(c)}{q_{2}(c) q_{3}(c)}, d=-q_{3}(c), f=-q_{4}(c), g=-a-p_{4} \text { and } h=\frac{p_{2}+c p_{4}}{q_{2}(c)} .
$$

Case 2. $p_{4}=p_{2}=p_{0}=0$. Take $a=1$ and choose any $c \neq 0$ with $q_{4}(c) \neq 0$. It is then possible to choose $h$ such that $1+c+p_{3}-h q_{4}(c) \neq 0$. Then take

$$
b=\frac{1+c+p_{3}-h q_{4}(c)}{q_{4}(c)}, d=f=-q_{4}(c) \text { and } g=-1 .
$$

Note that in each case the above choices give nonzero values for $a, b, c, d, f, g$ and $h$. It is straightforward to verify that these choices yield $p_{A}(x)=p(x)$.

While Proposition 2.7 shows immediately that $\mathcal{L}$ is not a SAP, the following corollary shows that $\mathcal{L}$ allows most refined inertias.

Corollary 2.8. Let $p(x)$ be a monic polynomial of degree 5 with real coefficients. If $p(x)$ has a zero on the imaginary axis, then $\mathcal{L}$ allows characteristic polynomial $p(x)$.

Proof. Suppose $p(x)=x^{5}+p_{4} x^{4}+p_{3} x^{3}+p_{2} x^{2}+p_{1} x+p_{0}$ has a zero $i \omega$ for some real $\omega$. Then

$$
0=p(i \omega)=i \omega^{5}+p_{4} \omega^{4}-i p_{3} \omega^{3}-p_{2} \omega^{2}+i p_{1} \omega+p_{0}
$$

and so the real part of the right-hand side must be zero, i.e. $p_{4} \omega^{4}-p_{2} \omega^{2}+p_{0}=0$. Therefore if $p_{0} \neq 0$, then $p_{4}$ and $p_{2}$ cannot both be zero, so $\mathcal{L}$ allows characteristic polynomial $p(x)$ by Proposition 2.7 .

THEOREM 2.9. The irreducible zero-nonzero pattern $\mathcal{L}$ of order 5 is an rIAP but not a SAP.

Proof. As already noted, Proposition 2.7 shows that $\mathcal{L}$ is not a SAP. To show that $\mathcal{L}$ is in fact an rIAP, first note that for $k \in\{0,1, \ldots, 5\}$ the polynomial $(x-1)^{k}(x+1)^{5-k}$ has a nonzero coefficient of $x^{4}$, and thus, by Proposition 2.7, 
is allowed as a characteristic polynomial by $\mathcal{L}$. Hence, $\mathcal{L}$ allows any refined inertia of the form $(k, 5-k, 0,0)$. By Corollary 2.8 , any refined inertia not of this form is also allowed by $\mathcal{L}$.

Note that, by Theorem 2.5, the pattern $\mathcal{L}$ is a smallest irreducible zero-nonzero pattern that is an rIAP while failing to be a SAP. Moreover, for any given refined inertia allowed by $\mathcal{L}$, or more generally for any given characteristic polynomial allowed by $\mathcal{L}$, the proof of Proposition 2.7 yields a constructive procedure for determining a matrix with pattern $\mathcal{L}$ having the given refined inertia or characteristic polynomial.

3. Refined inertia and reducible zero-nonzero patterns. Theorem 2.5 shows that an irreducible zero-nonzero pattern of order 4 or less that is an rIAP must be a SAP. The following proposition provides an analogous statement for reducible patterns.

Proposition 3.1. For $n \leq 5$, a reducible zero-nonzero pattern of order $n$ is an $I A P$ if and only if it is an rIAP if and only if it is a SAP.

Proof. If a pattern is a SAP, then it is obviously an rIAP (and therefore an IAP). For the converse, let $\mathcal{A}$ be a reducible zero-nonzero pattern of order $n \leq 5$. Then there exist zero-nonzero patterns of strictly smaller order, say $\mathcal{A}_{1}$ and $\mathcal{A}_{2}$, such that the characteristic polynomials (and hence the refined inertias) allowed by $\mathcal{A}$ are precisely those allowed by $\mathcal{A}_{1} \oplus \mathcal{A}_{2}$. Thus, it suffices to show that if $\mathcal{A}_{1} \oplus \mathcal{A}_{2}$ is an rIAP, then it is a SAP.

Let $\mathcal{A}^{\prime}=\mathcal{A}_{1} \oplus \mathcal{A}_{2}$. It is clear that if $\mathcal{A}^{\prime}$ has a summand of order 1 , then it is not an IAP. Hence, without loss of generality $\mathcal{A}_{1}$ has order 2 and $\mathcal{A}_{2}$ has order 3. Note that for $\mathcal{A}^{\prime}$ to allow the inertias $(5,0,0)$ and $(0,0,5), \mathcal{A}_{1}$ must allow the inertias $(2,0,0)$ and $(0,0,2)$, and $\mathcal{A}_{2}$ must allow the inertias $(3,0,0)$ and $(0,0,3)$. Thus $\mathcal{A}_{1}$ is a SAP by [10, Corollary 2] and $\mathcal{A}_{2}$ is a SAP by [10, Theorem 3]. Hence, $\mathcal{A}^{\prime}$ is the direct sum of two SAPs, only one of which has odd order. Such a pattern must be a SAP (see e.g., [6, Proposition 2.1]).

DeFinition 3.2. Let

$$
\mathcal{P}=\left[\begin{array}{llll}
* & * & 0 & 0 \\
* & * & * & 0 \\
0 & 0 & 0 & * \\
* & 0 & * & 0
\end{array}\right]
$$

This irreducible zero-nonzero pattern was introduced in [4] where it was shown that $\mathcal{P}$ fails to allow the inertia $(1,0,3)$. In particular, $\mathcal{P}$ is not an rIAP. The following proposition completely characterizes the refined inertias allowed by $\mathcal{P}$. 
Proposition 3.3. The irreducible zero-nonzero pattern $\mathcal{P}$ allows all refined inertias except for $(2,0,2,0),(2,0,0,2),(1,0,3,0)$ and $(1,0,1,2)$ and their reversals.

Proof. By Theorem 1.1, there are 14 (excluding reversals) refined inertias to verify. Without loss of generality a matrix $A$ with zero-nonzero pattern $\mathcal{P}$ is of the form

$$
A=\left[\begin{array}{llll}
a & 1 & 0 & 0 \\
b & c & 1 & 0 \\
0 & 0 & 0 & 1 \\
d & 0 & e & 0
\end{array}\right]
$$

for some nonzero real numbers $a, b, c, d$ and $e$. The characteristic polynomial of $A$ is

$$
p_{A}(x)=x^{4}-(a+c) x^{3}+(a c-b-e) x^{2}+e(a+c) x+(e(b-a c)-d) .
$$

Note that in $p_{A}(x)$ the coefficient of $x$ is zero if and only if the coefficient of $x^{3}$ is zero. Thus $A$ cannot have refined inertia $(2,0,2,0)$ or $(1,0,3,0)$.

Now suppose the refined inertia of $A$ is either $(1,0,1,2)$ or $(2,0,0,2)$. Then

$$
p_{A}(x)=\left(x^{2}+\alpha x+\beta\right)\left(x^{2}+\gamma\right)=x^{4}+\alpha x^{3}+(\beta+\gamma) x^{2}+\alpha \gamma x+\beta \gamma
$$

for some real numbers $\alpha, \beta$ and $\gamma$ where, in particular, $\alpha=-(a+c)$ cannot be zero. Comparison of the ratio of the coefficient of $x$ to that of $x^{3}$ in each of (3.2) and (3.3) yields $\gamma=-e$. Equating the coefficients of $x^{2}$ yields $\beta+\gamma=a c-b-e=a c-b+\gamma$ and hence $\beta=a c-b$. Finally, equating the constant terms gives

$$
\beta \gamma=e(b-a c)-d=(-\gamma)(-\beta)-d=\beta \gamma-d .
$$

But this implies that $d=0$, a contradiction. Hence $A$ cannot have refined inertia $(1,0,1,2)$ or $(2,0,0,2)$.

The following chart summarizes the refined inertias possible for a matrix with pattern $\mathcal{P}$. For each refined inertia allowed by $\mathcal{P}$, we provide a vector $(a, b, c, d, e)$ of values for the entries of $A$ in (3.1) such that $A$ has this refined inertia. 


\begin{tabular}{|c|c|c|c|c|c|c|}
\hline Refined inertia & Allowed by $\mathcal{P}$ & $(a$, & $b$, & $c$, & $d$, & $e)$ \\
\hline$(0,4,0,0)$ & yes & $(1$, & -10 & -5 & 4, & 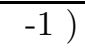 \\
\hline$(3,1,0,0)$ & yes & $(3$, & -14 & -4 & -8 & 5) \\
\hline$(0,3,1,0)$ & yes & $(1$, & $-\frac{20}{3}$ & -4 & $\frac{8}{9}$, & $\left.-\frac{1}{3}\right)$ \\
\hline$(2,2,0,0)$ & yes & $(1$, & $\frac{1}{2}$ & -1 & 1 & $\left.\frac{1}{2}\right)$ \\
\hline$(2,1,1,0)$ & yes & $(1$, & $\frac{1}{2}$, & 3 & $-\frac{15}{4}$ & $\left.\frac{3}{2}\right)$ \\
\hline$(2,0,2,0)$ & no & & & & & \\
\hline$(2,0,0,2)$ & no & & & & & \\
\hline$(1,1,2,0)$ & yes & $(1$, & -5 & -1 & -20 & 5) \\
\hline$(1,1,0,2)$ & yes & $(1$, & -2 , & -1 & 1, & 1 ) \\
\hline$(1,0,3,0)$ & no & & & & & \\
\hline$(1,0,1,2)$ & no & & & & & \\
\hline$(0,0,4,0)$ & yes & $(1$, & 1, & -1 & -4 & $-2)$ \\
\hline$(0,0,2,2)$ & yes & $(1$, & -3 & -1 & -2 & 1 ) \\
\hline$(0,0,0,4)$ & yes & $(1$, & -4 & -1 & -4 & 1) \\
\hline
\end{tabular}

The following theorem gives a reducible pattern of order 6 that is an rIAP despite failing to be a SAP. By Proposition 3.1 it is a smallest such example.

THEOREM 3.4. The reducible zero-nonzero pattern $\mathcal{P} \oplus \mathcal{T}_{2}$ of order 6 is an rIAP but not a $S A P$.

Proof. In Appendix A each refined inertia (excluding reversals) is expressed as a sum of two refined inertias, the first allowed by $\mathcal{P}$ and the second allowed by $\mathcal{T}_{2}$. Hence, $\mathcal{P} \oplus \mathcal{T}_{2}$ is an rIAP.

On the other hand, $\mathcal{P} \oplus \mathcal{T}_{2}$ is not a SAP, as it does not allow the characteristic polynomial

$$
x^{6}+x^{3}=x^{3}\left(x^{3}+1\right)=x^{3}(x+1)\left(x^{2}-x+1\right),
$$

since otherwise this polynomial would factor as $p(x) t(x)$, where $p(x)$ is a characteristic polynomial allowed by $\mathcal{P}$ and $t(x)$ is a characteristic polynomial allowed by $\mathcal{T}_{2}$. This gives rise to the following exhaustive list of cases.

Case 1: If $t(x)=x^{2}$ then $p(x)=x(x+1)\left(x^{2}-x+1\right)=x^{4}+x$.

Case 2: If $t(x)=x(x+1)$ then $p(x)=x^{2}\left(x^{2}-x+1\right)=x^{4}-x^{3}+x^{2}$.

Case 3: If $t(x)=x^{2}-x+1$ then $p(x)=x^{3}(x+1)=x^{4}+x^{3}$.

As $\mathcal{P}$ allows only characteristic polynomials with coefficients of $x$ and $x^{3}$ either both zero or both nonzero, none of these cases is possible. Hence, $\mathcal{P} \oplus \mathcal{T}_{2}$ is not a SAP. $\square$

In $[3,10]$ it is shown that a direct sum of two patterns may be an IAP even if neither of the summands is an IAP. This is shown in [3] both for sign patterns and for 
zero-nonzero patterns. Our next goal is to show that the analogous statement holds for rIAPs in the zero-nonzero case.

LEMma 3.5. Let $\mathcal{A}$ be a zero-nonzero pattern of order 4 allowing all of the refined inertias $(4,0,0,0),(0,0,4,0),(0,0,0,4),(0,0,2,2)$ and $(2,2,0,0)$. Then $\mathcal{A} \oplus \mathcal{M}$ is an $r I A P$.

Proof. In Appendix B, each refined inertia possible for a pattern of order 8 (excluding reversals) is expressed as a sum of two refined inertias, where the first is allowed by $\mathcal{A}$ and the second is allowed by $\mathcal{M}$ by Proposition 2.3. $\square$

THEOREM 3.6. The reducible zero-nonzero pattern $\mathcal{P} \oplus \mathcal{M}$ of order 8 is an rIAP with neither of its summands an rIAP.

Proof. This follows immediately from Lemma 3.5 and Proposition 3.3.

Note that $\mathcal{P} \oplus \mathcal{M}$ in the statement of Theorem 3.6 cannot be replaced by any direct sum with smaller order. To see this, consider some pattern $\mathcal{A}=\mathcal{A}_{1} \oplus \mathcal{A}_{2}$ of order $n \leq 7$ and suppose $\mathcal{A}$ is an rIAP. Then neither of the $\mathcal{A}_{i}$ may have order 1 . Hence, at least one of them must have order $k$ for $k=2$ or $k=3$. Say this is true of $\mathcal{A}_{1}$. Since $\mathcal{A}$ allows the inertias $(n, 0,0)$ and $(0,0, n)$, it follows that $\mathcal{A}_{1}$ allows the inertias $(k, 0,0)$ and $(0,0, k)$. But by [10, Corollary 2] (for $k=2)$ or [10, Theorem 3] (for $k=3$ ) this implies that $\mathcal{A}_{1}$ is a SAP, and hence an rIAP. Therefore $\mathcal{A}_{1} \oplus \mathcal{A}_{2}$ cannot replace $\mathcal{P} \oplus \mathcal{M}$ in the statement of Theorem 3.6.

Note also that $\mathcal{P} \oplus \mathcal{M}$ is not a $\mathrm{SAP}$, as it does not allow the characteristic polynomial $x^{2}\left(x^{2}-2 x+2\right)^{3}$. To justify this claim, we refer to the proof of $[6$, Proposition 2.2 (case 3)] in which it is shown that a particular signing of $\mathcal{M}$ does not allow any characteristic polynomial with two non-real zeros if the other two are zero. In fact this proof shows that the same is true of the zero-nonzero pattern $\mathcal{M}$. Thus for $x^{2}\left(x^{2}-2 x+2\right)^{3}$ to factor as the product of a characteristic polynomial allowed by $\mathcal{P}$ and one allowed by $\mathcal{M}$, the factor allowed by $\mathcal{M}$ must be $\left(x^{2}-2 x+2\right)^{2}$. But this leaves $\mathcal{P}$ to account for the factor $x^{2}\left(x^{2}-2 x+2\right)$, which by Proposition 3.3 it cannot, as $\mathcal{P}$ does not allow the refined inertia $(2,0,2,0)$.

4. Reducible spectrally arbitrary patterns. It is known that the direct sum of two SAPs need not be a SAP. In fact, it is shown in [6, Proposition 2.1] that when two or more summands are of odd order, a direct sum cannot be a SAP. On the other hand, a particular signing of $\mathcal{M} \oplus \mathcal{T}_{2}$ is shown in [6, Proposition 2.4] to be a SAP, despite one of its summands (the signing of $\mathcal{M}$ ) failing to be itself a SAP. The natural question has been raised (see $[2,3])$ as to whether a direct sum of two non-SAPs could be a SAP. The principal aim of this section is to show that this is indeed possible for zero-nonzero patterns. To this end, we obtain for the pattern $\mathcal{L}$ introduced in Definition 2.6 an analog of Lemma 2.2. 
LEMMA 4.1. Let $p(x)$ be a monic polynomial of degree 5 with real coefficients. Each of the following conditions is sufficient for $\mathcal{L}$ to allow characteristic polynomial $p(x)$.

(i) $p(0)=0$.

(ii) The sum of the zeros of $p(x)$ is nonzero.

(iii) $p(x)$ has only real zeros, one of which has multiplicity three or more.

(iv) $p(x)$ has two irreducible quadratic factors with identical coefficients of $x$.

Proof. The sufficiency of each of conditions (i) and (ii) follows immediately from Proposition 2.7 .

Assume $p(x)$ satisfies condition (iii). If $p(0)=0$, then (i) applies and the proof is complete. If not, then $p(x)=(x-a)^{3}(x-b)(x-c)$ for some nonzero real numbers $a$, $b$ and $c$, in which case the coefficient of $x^{4}$ in $p(x)$ is $-(3 a+b+c)$ while the coefficient of $x^{2}$ is $-a\left(a^{2}+3 a b+3 a c+3 b c\right)$. If $\mathcal{L}$ does not allow characteristic polynomial $p(x)$, then by Proposition 2.7 both of these coefficients are zero. In particular, the zero coefficient of $x^{4}$ gives $a=-\frac{b+c}{3}$ so that the zero coefficient of $x^{2}$ requires

$$
0=a^{2}+3 a b+3 a c+3 b c=\frac{-8(b+c)^{2}}{9}+3 b c .
$$

This implies that $(b+c)^{2}=\frac{27}{8} b c$, which is impossible as $(b+c)^{2} \geq 4 b c$ and $b c$ is nonzero.

Finally, suppose that $p(x)$ satisfies condition (iv). Then

$$
\begin{aligned}
p(x)= & (x+a)\left(x^{2}+b x+c\right)\left(x^{2}+b x+d\right) \\
= & x^{5}+(a+2 b) x^{4}+\left(2 a b+b^{2}+c+d\right) x^{3} \\
& +\left(a b^{2}+a c+a d+b c+b d\right) x^{2}+(a b c+a b d+c d) x+(a c d)
\end{aligned}
$$

for some nonzero real numbers $a, b, c$ and $d$ such that $x^{2}+b x+c$ and $x^{2}+b x+d$ are irreducible. Note that the irreducibility of these quadratic factors requires that $c$ and $d$ be positive. If either the coefficient of $x^{4}$ or that of $x^{2}$ is nonzero, then by Proposition 2.7 the proof is complete. Otherwise, the zero coefficient of $x^{4}$ requires $a=-2 b$ and hence

$p(x)=x^{5}+\left(-3 b^{2}+c+d\right) x^{3}+\left(-2 b^{3}-b c-b d\right) x^{2}+\left(-2 b^{2} c-2 b^{2} d+c d\right) x+(-2 b c d)$

so that the zero coefficient of $x^{2}$ requires $-2 b^{3}-b c-b d=-b\left(2 b^{2}+c+d\right)=0$. But $2 b^{2}+c+d>0$, as $c$ and $d$ are positive. Hence it must be that $b=0$, but then the constant term of $p(x)$ is zero, and so by Proposition 2.7 the proof is complete in this case as well.

THEOREM 4.2. The reducible pattern $\mathcal{L} \oplus \mathcal{M}$ of order 9 is a SAP with neither of its summands a SAP. 
Proof. Let $\Pi_{\mathcal{L}}$ be the set of polynomials allowed as characteristic polynomials by $\mathcal{L}$ and define $\Pi_{\mathcal{M}}$ similarly. Let $p(x)$ be an arbitrary monic polynomial of degree 9 with real coefficients. We must show that $\mathcal{L} \oplus \mathcal{M}$ allows characteristic polynomial $p(x)$. It suffices to express $p(x)$ as the product of a polynomial in $\Pi_{\mathcal{L}}$ and one in $\Pi_{\mathcal{M}}$. The proof breaks down naturally according to the number of irreducible quadratic factors of $p(x)$.

Case 1. Suppose $p(x)$ has no irreducible quadratic factor. That is, $p(x)$ has only real zeros. Then

$$
p(x)=g_{1} g_{2} g_{3} g_{4} g_{5} g_{6} g_{7} g_{8} g_{9}
$$

where each $g_{i}=x-r_{i}$ for some real number $r_{i}$. If at least two of the $r_{i}$ are zero, say $r_{1}=r_{5}=0$, then $g_{1} g_{2} g_{3} g_{4} \in \Pi_{\mathcal{M}}$ by Lemma 2.2(ii) while $g_{5} g_{6} g_{7} g_{8} g_{9} \in \Pi_{\mathcal{L}}$ by Lemma 4.1(i).

Now suppose exactly one of the $r_{i}$ is zero, say $r_{1}=0$. Then, as no other $r_{i}$ is zero, there must be some four among $g_{2}, \ldots, g_{9}$ whose product has a positive constant term. Assume this is true of $g_{6}, \ldots, g_{9}$. Then $g_{6} g_{7} g_{8} g_{9} \in \Pi_{\mathcal{M}}$ by Lemma 2.2(i), while $g_{1} g_{2} g_{3} g_{4} g_{5} \in \Pi_{\mathcal{L}}$ by Lemma 4.1(i).

Finally, suppose no $r_{i}$ is zero. Then certainly there are some five of the $r_{i}$ with the same sign. Assume this is true of $r_{1}, \ldots, r_{5}$. Then any product of four factors from $g_{1}, \ldots, g_{5}$ has a positive constant term and therefore is in $\Pi_{\mathcal{M}}$. Hence, if $g_{i} g_{6} g_{7} g_{8} g_{9} \in$ $\Pi_{\mathcal{L}}$ for any $i \in\{1, \ldots, 5\}$, then the proof is complete. If not, then by Lemma 4.1(ii) the zeros of each of these products must sum to zero. That is, $r_{i}=-r_{6}-r_{7}-r_{8}-r_{9}$ for $i=1, \ldots, 5$. Thus, letting $r=-r_{6}-r_{7}-r_{8}-r_{9}$ yields

$$
p(x)=(x-r)^{5} g_{6} g_{7} g_{8} g_{9} .
$$

Clearly some pair from $r_{6}, \ldots, r_{9}$ must have the same sign, say $r_{6}$ and $r_{7}$. Then $(x-r)^{2} g_{6} g_{7} \in \Pi_{\mathcal{M}}$ by Lemma 2.2(i) while $(x-r)^{3} g_{8} g_{9} \in \Pi_{\mathcal{L}}$ by Lemma 4.1(iii).

Case 2. Suppose $p(x)$ has exactly one irreducible quadratic factor. Then

$$
p(x)=g_{1} g_{2} g_{3} g_{4} g_{5} g_{6} g_{7} f_{1}
$$

where each $g_{i}=x-r_{i}$ for some real number $r_{i}$ and $f_{1}$ is a quadratic that is irreducible and hence must have a positive constant term.

First suppose some $r_{i}$ is zero, say $r_{1}=0$. Among $g_{2}, \ldots, g_{7}$ there must be some four factors whose product does not have a negative constant term, and so is in $\Pi_{\mathcal{M}}$ by Lemma 2.2. Then, since $r_{1}=0$, it follows from Lemma 4.1(i) that the product of $f_{1}$ and the remaining $g_{i}$ is in $\Pi_{\mathcal{L}}$.

Now assume that no $r_{i}$ is zero. Suppose some five of the $r_{i}$ are of the same sign, say $r_{1}, \ldots, r_{5}$. Then the product of any four of $g_{1}, \ldots, g_{5}$ is in $\Pi_{\mathcal{M}}$ by Lemma 2.2(i). 
Thus if $g_{i} g_{6} g_{7} f_{1} \in \Pi_{\mathcal{L}}$ for any $i \in\{1, \ldots, 5\}$, then the proof is complete. If not, then Lemma 4.1(ii) implies that $r_{1}=r_{2}=\cdots=r_{5}$. But then $g_{1} g_{2} g_{3} g_{6} g_{7} \in \Pi_{\mathcal{L}}$ by Lemma 4.1(iii) while $g_{4} g_{5} f_{1} \in \Pi_{\mathcal{M}}$ by Lemma 2.2(i).

Now suppose that four of the $r_{i}$, say $r_{1}, \ldots, r_{4}$, have one sign while $r_{5}, \ldots, r_{7}$ have the opposite sign. Then $g_{1} g_{2} g_{i} g_{j} \in \Pi_{\mathcal{M}}$ for any $i$ and $j$ with $5 \leq i<j \leq 7$. Thus if $g_{i} g_{3} g_{4} f_{1} \in \Pi_{\mathcal{L}}$ for any $i \in\{5,6,7\}$, then the proof is complete. If not, then Lemma 4.1(ii) implies that $r_{5}=r_{6}=r_{7}$. But then $g_{3} g_{4} g_{5} g_{6} g_{7} \in \Pi_{\mathcal{L}}$ by Lemma 4.1(iii) while $g_{1} g_{2} f_{1} \in \Pi_{\mathcal{M}}$ by Lemma 2.2(i).

Case 3. Suppose $p(x)$ has exactly two irreducible quadratic factors. Then

$$
p(x)=g_{1} g_{2} g_{3} g_{4} g_{5} f_{1} f_{2}
$$

where each $g_{i}=x-r_{i}$ for some real number $r_{i}$ and each $f_{j}$ is a quadratic that is irreducible and hence must have a positive constant term.

If some $r_{i}$ is zero, say $r_{1}=0$, then $f_{1} f_{2} \in \Pi_{\mathcal{M}}$ by Lemma 2.2(i) while $g_{1} g_{2} g_{3} g_{4} g_{5} \in$ $\Pi_{\mathcal{L}}$ by Lemma 4.1(i). If no $r_{i}$ is zero then certainly some two $r_{i}$ have the same sign, say $r_{1}$ and $r_{2}$. Then $g_{1} g_{2} f_{1}$ and $g_{1} g_{2} f_{2}$ are in $\Pi_{\mathcal{M}}$ by Lemma 2.2(i). Thus if either $g_{3} g_{4} g_{5} f_{1}$ or $g_{3} g_{4} g_{5} f_{2}$ is in $\Pi_{\mathcal{L}}$, then the proof is complete. If not, then it follows from Lemma 4.1(ii) that $f_{1}$ and $f_{2}$ have equal coefficients of $x$. But then $g_{i} f_{1} f_{2} \in \Pi_{\mathcal{L}}$ for each $g_{i}$ by Lemma 4.1(iv), and it suffices to show that some product of four of the $g_{i}$ is in $\Pi_{\mathcal{M}}$. But by Lemma 2.2(i) this fails to hold only if every such product has a negative constant term, and it is easy to check that this is combinatorially impossible.

Case 4. Suppose $p(x)$ has exactly three irreducible quadratic factors. Then

$$
p(x)=g_{1} g_{2} g_{3} f_{1} f_{2} f_{3}
$$

where each $g_{i}=x-r_{i}$ for some real number $r_{i}$ and each $f_{j}$ is a quadratic that is irreducible and hence must have a positive constant term.

If some $r_{i}$ is zero, say $r_{1}=0$, then $f_{1} f_{2} \in \Pi_{\mathcal{M}}$ by Lemma 2.2(i) while $g_{1} g_{2} g_{3} f_{3} \in$ $\Pi_{\mathcal{L}}$ by Lemma 4.1(i). If no $r_{i}$ is zero then certainly some two $r_{i}$ have the same sign, say $r_{1}$ and $r_{2}$. Then $g_{1} g_{2} f_{1}$ and $g_{1} g_{2} f_{2}$ are in $\Pi_{\mathcal{M}}$ by Lemma 2.2(i). Thus if either $g_{3} f_{2} f_{3}$ or $g_{3} f_{1} f_{3}$ is in $\Pi_{\mathcal{L}}$, then the proof is complete. If not, then it follows from Lemma 4.1(ii) that $f_{1}$ and $f_{2}$ have equal coefficients of $x$. But then $g_{3} f_{1} f_{2} \in \Pi_{\mathcal{L}}$ by Lemma 4.1(iv) while $g_{1} g_{2} f_{3} \in \Pi_{\mathcal{M}}$ by Lemma 2.2(i).

Case 5. Suppose $p(x)$ has exactly four irreducible quadratic factors. Then

$$
p(x)=g_{1} f_{1} f_{2} f_{3} f_{4}
$$

where $g_{1}=x-r_{1}$ for some real number $r_{1}$ and each $f_{j}$ is a quadratic that is irreducible and hence must have a positive constant term. 
By Lemma 2.2(i) both $f_{1} f_{3}$ and $f_{1} f_{4}$ are in $\Pi_{\mathcal{M}}$. Thus if either $g_{1} f_{2} f_{4}$ or $g_{1} f_{2} f_{3}$ is in $\Pi_{\mathcal{L}}$, then the proof is complete. If not, then it follows from Lemma 4.1(ii) that $f_{3}$ and $f_{4}$ have equal coefficients of $x$. But then $g_{1} f_{3} f_{4} \in \Pi_{\mathcal{L}}$ by Lemma 4.1(iv) while $f_{1} f_{2} \in \Pi_{\mathcal{M}}$ by Lemma $2.2(\mathrm{i})$.

Note that the above proof of Theorem 4.2 uses only properties of $\mathcal{M}$ following from Lemma 2.2, and this lemma remains true for the particular signing of $\mathcal{M}$ analyzed in $[6]$.

5. Concluding remarks. The pattern $\mathcal{L}$ is a superpattern of the zero-nonzero pattern $\mathcal{K}$ introduced in [10]. In particular, $\mathcal{K}$ is the pattern that results from replacing the $(2,5)$ entry of $\mathcal{L}$ with 0 . In $[10]$ it was shown that $\mathcal{K}$ is not an IAP but $\mathcal{K} \oplus \mathcal{K}$ is an IAP. As Theorem 2.9 shows, the pattern $\mathcal{L}$ provides a negative answer to the zerononzero analog of question (b) of [9, page 174] that was asked in the sign pattern case. This question, which remains open for both an irreducible sign pattern and a direct sum of sign patterns, asks if a refined inertially arbitrary sign pattern is necessarily spectrally arbitrary. In addition, the question remains open as to whether there exists a direct sum of two sign patterns that is spectrally arbitrary with neither summand being spectrally arbitrary (cf. Theorem 4.2), although it is known [6, Proposition $2.4]$ that a direct sum may be spectrally arbitrary if only one of the summands is spectrally arbitrary.

Acknowledgements. The research of Dale Olesky and Pauline van den Driessche is partially supported by NSERC Discovery grants. These authors thank Michael Rempel for initial investigations on refined inertias of sign patterns.

\section{REFERENCES}

[1] T. Britz, J. J. McDonald, D. D. Olesky, and P. van den Driessche. Minimal spectrally arbitrary sign patterns. SIAM J. Matrix Anal. Appl., 26(1):257-271, 2004.

[2] M. Catral, D. D. Olesky, and P. van den Driessche. Allow problems concerning spectral properties of sign pattern matrices: a survey. Linear Algebra Appl., 430(11-12):3080-3094, 2009.

[3] M. S. Cavers. On reducible matrix patterns. Linear Multilinear Algebra, 58(2):257-267, 2010.

[4] M. S. Cavers and K. N. Vander Meulen. Inertially arbitrary nonzero patterns of order 4. Electron. J. Linear Algebra, 16:30-43, 2007.

[5] L. Corpuz and J. J. McDonald. Spectrally arbitrary zero-nonzero patterns of order 4. Linear Multilinear Algebra, 55(3):249-273, 2007.

[6] L. M. DeAlba, I. R. Hentzel, L. Hogben, J. J. McDonald, R. Mikkelson, O. Pryporova, B. Shader, and K. N. Vander Meulen. Spectrally arbitrary patterns: reducibility and the $2 n$ conjecture for $n=5$. Linear Algebra Appl., 423(2-3):262-276, 2007.

[7] J. H. Drew, C. R. Johnson, D. D. Olesky, and P. van den Driessche. Spectrally arbitrary patterns. Linear Algebra Appl., 308(1-3):121-137, 2000. 
[8] I.-J. Kim, J. J. McDonald, D. D. Olesky, and P. van den Driessche. Inertias of zero-nonzero patterns. Linear Multilinear Algebra, 55(3):229-238, 2007.

[9] I.-J. Kim, D. D. Olesky, B. L. Shader, P. van den Driessche, H. van der Holst, and K. N. Vander Meulen. Generating potentially nilpotent full sign patterns. Electron. J. Linear Algebra, 18:162-175, 2009.

[10] I.-J. Kim, D. D. Olesky, and P. van den Driessche. Critical sets of inertias for matrix patterns. Linear Multilinear Algebra, 57(3):293-306, 2009.

[11] D. O. Logofet. Matrices and Graphs: Stability Problems in Mathematical Ecology, CRC Press, Boca Raton, FL, 1992. 
Appendix A. List of refined inertias allowed by $\mathcal{P} \oplus \mathcal{T}_{2}$ for Theorem 3.4.

$\begin{array}{lll}(6,0,0,0)=(4,0,0,0)+(2,0,0,0) & (3,1,0,2)=(3,1,0,0)+(0,0,0,2) & (1,1,4,0)=(0,0,4,0)+(1,1,0,0) \\ (5,1,0,0)=(4,0,0,0)+(1,1,0,0) & (3,0,3,0)=(3,0,1,0)+(0,0,2,0) & (1,1,2,2)=(0,0,2,2)+(1,1,0,0) \\ (5,0,1,0)=(4,0,0,0)+(1,0,1,0) & (3,0,1,2)=(3,0,1,0)+(0,0,0,2) & (1,1,0,4)=(0,0,0,4)+(1,1,0,0) \\ (4,2,0,0)=(4,0,0,0)+(0,2,0,0) & (2,2,2,0)=(2,2,0,0)+(0,0,2,0) & (1,0,5,0)=(0,0,4,0)+(1,0,1,0) \\ (4,1,1,0)=(4,0,0,0)+(0,1,1,0) & (2,2,0,2)=(2,2,0,0)+(0,0,0,2) & (1,0,3,2)=(0,0,2,2)+(1,0,1,0) \\ (4,0,2,0)=(4,0,0,0)+(0,0,2,0) & (2,1,3,0)=(2,1,1,0)+(0,0,2,0) & (1,0,1,4)=(0,0,0,4)+(1,0,1,0) \\ (4,0,0,2)=(4,0,0,0)+(0,0,0,2) & (2,1,1,2)=(2,1,1,0)+(0,0,0,2) & (0,0,6,0)=(0,0,4,0)+(0,0,2,0) \\ (3,3,0,0)=(3,1,0,0)+(0,2,0,0) & (2,0,4,0)=(0,0,4,0)+(2,0,0,0) & (0,0,4,2)=(0,0,4,0)+(0,0,0,2) \\ (3,2,1,0)=(3,1,0,0)+(0,1,1,0) & (2,0,2,2)=(0,0,2,2)+(2,0,0,0) & (0,0,2,4)=(0,0,0,4)+(0,0,2,0) \\ (3,1,2,0)=(3,1,0,0)+(0,0,2,0) & (2,0,0,4)=(0,0,0,4)+(2,0,0,0) & (0,0,0,6)=(0,0,0,4)+(0,0,0,2)\end{array}$

\section{Appendix B. List of refined inertias allowed by $\mathcal{A} \oplus \mathcal{M}$ for Lemma 3.5.}

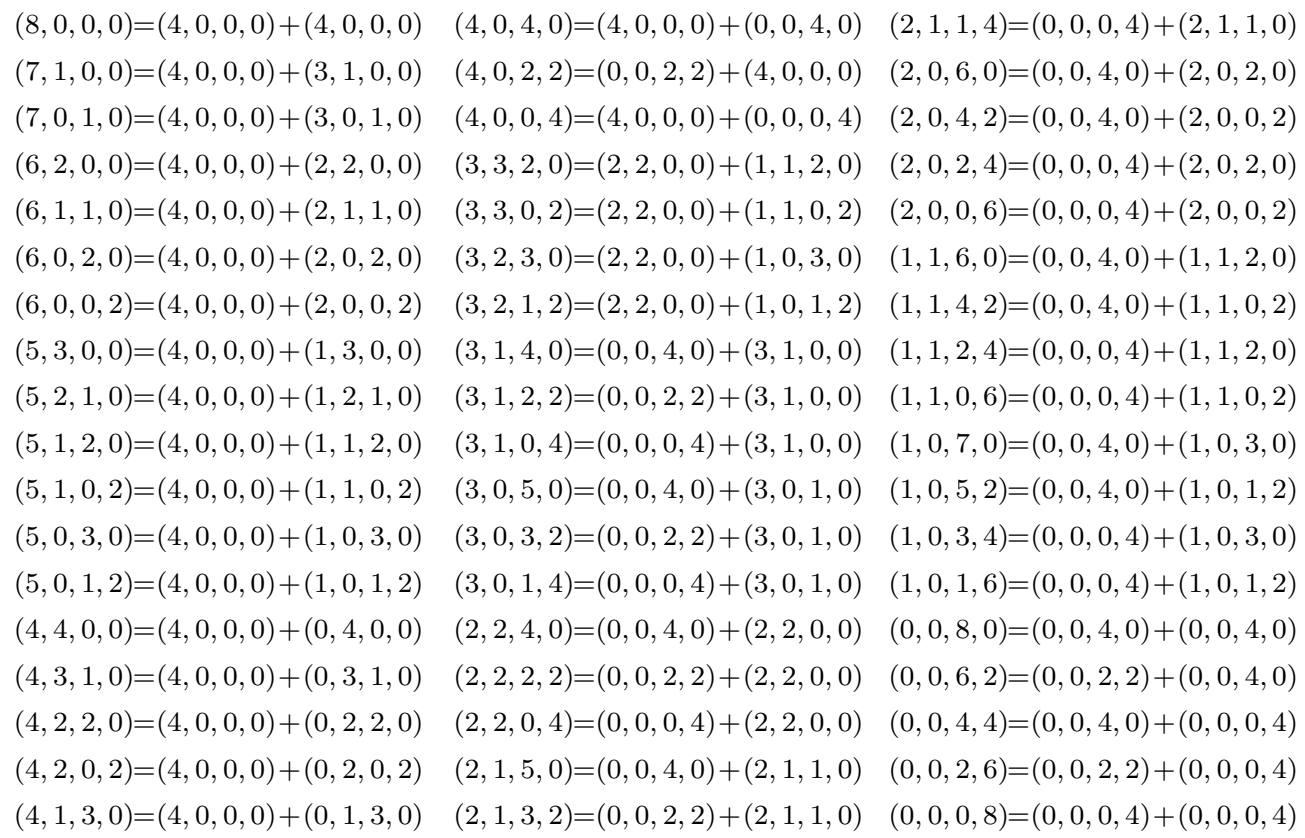

